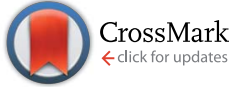

Cite this: RSC Adv., 2017, 7, 8098

Received 5th December 2016 Accepted 10th January 2017

DOI: 10.1039/c6ra27761f

www.rsc.org/advances

\section{Anisotropic phonon transport and lattice thermal conductivities in tin dichalcogenides $\mathrm{SnS}_{2}$ and $\mathrm{SnSe}_{2}$}

\begin{abstract}
Haifeng Wang, *a Yan Gao and Gang Liu ${ }^{b}$
In recent years, layered semiconductor tin dichalcogenides, $\operatorname{SnX} X_{2}(X=S$ and $\mathrm{Se}$ ), have received great attention owing to their wide applications in numerous fields. However, theoretical studies on their phonon transport properties and lattice thermal conductivities are very rare. Herein, we carry out comprehensive investigations on the phonon transport properties and heat transfer phenomena in tin dichalcogenides using first-principles calculations combined with the Boltzmann transport theory, and compare them with the recent popular thermoelectric materials SnS ( $\mathrm{SnSe}$ ) and widely studied typical TMD $\mathrm{MoS}_{2}\left(\mathrm{MoSe}_{2}\right)$. The obtained total thermal conductivities of $\mathrm{SnS}_{2}$ and $\mathrm{SnSe}_{2}$ agree well with the experimental measured values. The ultralow out-of-plane thermal conductivities of both materials may be useful for thermoelectric applications. The contributions of different phonon branches to the total lattice thermal conductivities are evaluated, and the results suggest that it would be difficult for alloying to reduce $\kappa_{\mathrm{L}}$ because the contribution of the optical branches is rather small compared with SnS and SnSe. Subsequently, we investigated the size dependence of the thermal conductivities and found that nanostructuring may be efficient to further reduce $\kappa_{\mathrm{L}}$ since the mean free paths of the dominant phonon modes are relatively long. This conclusion is consistent with recent experimental findings, where the $\kappa_{\mathrm{L}}$ of $\mathrm{SnS}_{2}$ was found to evidently decrease with a decrease in the thickness of $\mathrm{SnS}_{2}$ films. We believe that this phenomenon may also exist in $\mathrm{SnSe}_{2}$, which may demonstrate a much better thermoelectric performance than that of $\mathrm{SnS}_{2}$ because of its higher anharmonicity than that of $\mathrm{SnS}_{2}$, leading to much lower thermal conductivity.
\end{abstract}

\section{Introduction}

Transition metal dichalcogenides (TMDs) $\left(\mathrm{MX}_{2}\right.$, where $\mathrm{M}$ denotes a transition metal and $\mathrm{X}$ denotes a chalcogen) are currently attracting increasing interest as a group of mostly semiconducting two-dimensional (2D) and few-layer materials that complement other $2 \mathrm{D}$ systems, such as graphene $\mathrm{e}^{1,2}$ or h-BN. ${ }^{3,4}$ Among all the TMDs, tin dichalcogenides $\left(\mathrm{SnS}_{2}\right.$ and $\mathrm{SnSe}_{2}$ ) (hereafter denoted as $\mathrm{SnX}_{2}$ ) are more earth-abundant and environmentally-friendly. Recently, they have attracted much attention due to their great potential in a wide spectrum of applications, e.g., thin film solar cells, optoelectronic devices, field-effect transistors (FETs), lithium-ion batteries and portable electronic information storage devices. ${ }^{5-12}$ In spite of the large amount of research on their structural, electronic and optical properties ${ }^{11-16}$ only limited information is now available concerning the thermoelectric performances of $\mathrm{SnS}_{2}$ and $\mathrm{SnSe}_{2} \cdot{ }^{17-19}$ In the recent theoretical study by B. Sun et al.,${ }^{18}$ it was

${ }^{a}$ Department of Physics, College of Science, Shihezi University, Xinjiang 832003, China.E-mail:whfeng@shzu.edu.cn

${ }^{b}$ School of Physics and Engineering, Henan University of Science and Technology, Luoyang 471023, China shown that the peak $Z T$ can reach as high as 0.96 for $\mathrm{SnS}_{2}$ and 0.88 for $\mathrm{SnSe}_{2}$ at room temperature, which shows their potential for TE applications. Moreover, in another recent experimental study, ${ }^{19}$ the authors suggest that $\mathrm{SnS}_{2}$ nanosheets are negatively correlated thermoelectric materials with a negative correlation between electrical and thermal conductivity, that is, as the thickness of $\mathrm{SnS}_{2}$ decreases, $s$ increases, whereas $\kappa$ decreases. Naturally, this is an important finding since most TE materials demonstrate decreased thermal conductivity when their electrical conductivity increases. Although the lattice thermal conductivity is crucial to the thermoelectric performance of TE materials, to date, theoretical reports that discuss the lattice thermal conductivity of $\mathrm{SnX}_{2}$ are still lacking, with the exception of one recent study ${ }^{18}$ that considered the theoretically minimum lattice thermal conductivity $\left(\kappa_{\mathrm{min}}\right)$. On the other hand, several of the experimentally measured values of thermal conductivity differ enormously. ${ }^{19-23}$ Furthermore, a complete and comparative prediction and understanding of the underlying phonon transport properties of $\mathrm{SnS}_{2}$ and $\mathrm{SnSe}_{2}$ are key to expanding the range of their application in nanoelectronics, optoelectronics and thermoelectrics; however, thus far, the phonon transport properties of these two dichalcogenides are still less known. Therefore, it is necessary to give a detailed study of their 
phonon transport and accurately predict their intrinsic thermal conductivity. Another fact that should be noted is that the present materials have several similarities with $\mathrm{SnS}$ and SnSe, which have been recently found to display high thermoelectric efficiencies with great potential for the conversion of heat to electricity. ${ }^{24,25}$ For example, they all are constituted of Sn and S (Se) that tend to form weak atomic bonds and usually lead to strong anharmonicity. In addition, they all have anisotropic (orthorhombic) layered structures and the interplanar van der Waals forces usually bring ultralow out-of-plane thermal conductivity. Then, the question arises of whether $\mathrm{SnX}_{2}$ could perform well as thermal insulators similar to SnS and SnSe.

Herein, we conduct comprehensive investigations into the anisotropic phonon transport properties and intrinsic thermal conductivities of $\mathrm{SnX}_{2}$ based on first-principles calculations. A systematic study of the lattice dynamics are performed and related properties, including Born effective charge (BEC), dielectric constant (DC) and frequencies of optical modes, together with their irreducible representation are given. Then, the lattice thermal transports of both materials are calculated by solving the phonon Boltzmann transport equation. The obtained thermal conductivities of $\mathrm{SnS}_{2}$ and $\mathrm{SnSe}_{2}$ are close to the experimental measured values. The contributions of each phonon branch to the total thermal conductivities, phonon lifetimes and the dependence of thermal conductivities for both materials are evaluated, and the underlying mechanism governing their diverse thermal transport behavior are analyzed and discussed in detail.

\section{Computational details}

First-principles calculations were performed using the density functional theory as implemented in the Vienna $a b$ initio simulation package (VASP) ${ }^{26-28}$ with a plane wave energy of up to $500 \mathrm{eV}$ in the expansion of the electronic wave function. The local density approximation (LDA), ${ }^{29}$ which is typically used for layered structures where van der Waals interactions are important, was chosen as the exchange-correlation functional of DFT. The valence electron configurations considered in this calculation are $\mathrm{Sn}\left(4 \mathrm{~d}^{10} 5 \mathrm{~s}^{2} 5 \mathrm{p}^{2}\right), \mathrm{S}\left(3 \mathrm{~s}^{2} 3 \mathrm{p}^{4}\right)$, and Se $\left(4 \mathrm{~s}^{2} 4 \mathrm{p}^{4}\right)$. The internal coordinates and lattice constants were optimized until the Hellmann-Feynman forces acting on each atom became less than $10^{-3} \mathrm{eV}^{-1}$. The convergence for energy was chosen as $10^{-6} \mathrm{eV}$ between two steps, and a Monkhorst-Pack $k$-mesh of $7 \times 7 \times 5$ was used to sample the Brillouin zone in the structure optimization. Phonon frequencies and the corresponding thermodynamic properties were obtained using the PHONOPY ${ }^{30}$ program. Moreover, an iterative self-consistent method was used for solving the phonon Boltzmann transport equation to calculate the lattice thermal conductivity with the ShengBTE $^{31}$ code, which is based on the second-order (harmonic) and third-order (anharmonic) interatomic force constants (IFCs) combined with an iterative self-consistent algorithm to solve the BTE, and has also successfully predicted the thermal conductivities of many materials. ${ }^{32-38} \mathrm{We}$ used $4 \times 4 \times 2$ supercells containing 96 atoms with $3 \times 3 \times 3 k$ mesh for calculations of second-order and third-order IFCs for both $\mathrm{SnS}_{2}$ and $\mathrm{SnSe}_{2}$. In the calculations of the third-order IFCs, we considered third-order interatomic force constants up to the fourth shell of neighbors. Well-converged $20 \times 20 \times 11 q$ meshes were used in the calculation of the lattice thermal conductivity of $\mathrm{SnX}_{2}$.

\section{Results and discussion}

Tin dichalcogenides $\left(\mathrm{SnS}_{2}\right.$ and $\left.\mathrm{SnSe}_{2}\right)$ crystallize in the hexagonal closed packed $\mathrm{CdI}_{2}$-type structure with $P \overline{3} m 1$ symmetry (space group 164). They have three atoms in the unit cell, which extends over only one sandwich layer as seen in Fig. 1. The intralayer metal (Sn)-chalcogenide (S or $\mathrm{Se}$ ) bonds are predominantly covalent in nature, whereas the layers themselves are coupled by weak van der Waals bonds. The Sn atoms provide four electrons to fill the bonding states of $\mathrm{SnX}_{2}$ such that the oxidation states of the $\mathrm{Sn}$ and $\mathrm{S}$ (or Se) atoms are +4 and -2 , respectively. Our relaxed lattice constants resulting from LDA are $a=3.63 \AA$ and $c=5.71 \AA$ for $\mathrm{SnS}_{2}$, and $a=3.80 \AA$ and $c$ $=5.90 \AA$ for $\mathrm{SnSe}_{2}$, which agree well with previous ab initio calculations ${ }^{\mathbf{1 3}}$ but are slightly underestimated compared with the experimental results. ${ }^{39}$ This is reasonable because of the overbinding effect of LDA.

\subsection{Lattice dynamics properties}

The harmonic phonon dispersions of $\mathrm{SnX}_{2}$ as obtained from the force constants and considering the longitudinal opticaltransverse optical (LO-TO) splitting are shown in Fig. 2. The LO-TO splitting is predicted by incorporating the effects of long-range Coulomb interactions based on the Born effective charges and high-frequency dielectric constants calculated by first-principles. Our calculated Born effective charges and dielectric constants are illustrated in Table 1. It is worth noting that the ionic contribution to the dielectric constant of $\mathrm{SnS}_{2}$ along the out-of-plane ( $c$ axis) direction is very small (0.69), which indicates that $\mathrm{SnS}_{2}$ holds more two-dimensional characteristics than $\mathrm{SnSe}_{2}$. This is consistent with the fact that the out-of-plane thermal conductivity of $\mathrm{SnS}_{2}$ is much lower than that of $\mathrm{SnSe}_{2}$, which will be discussed later.

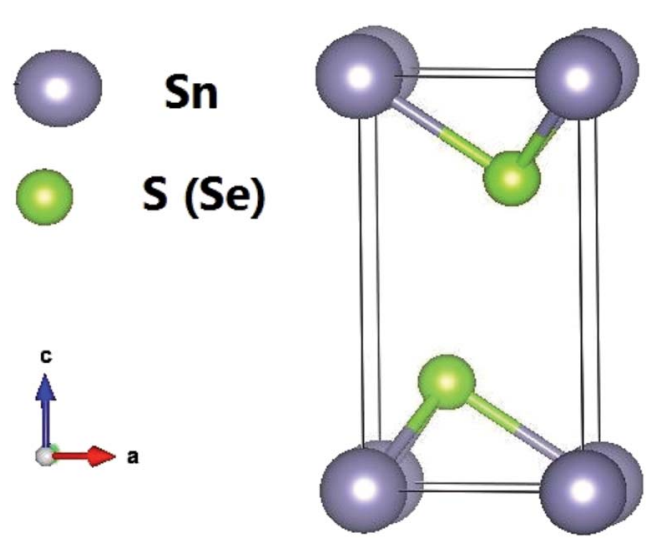

Fig. 1 Crystal structures of $\mathrm{SnX}_{2}(X=S$ and $\mathrm{Se})$. 

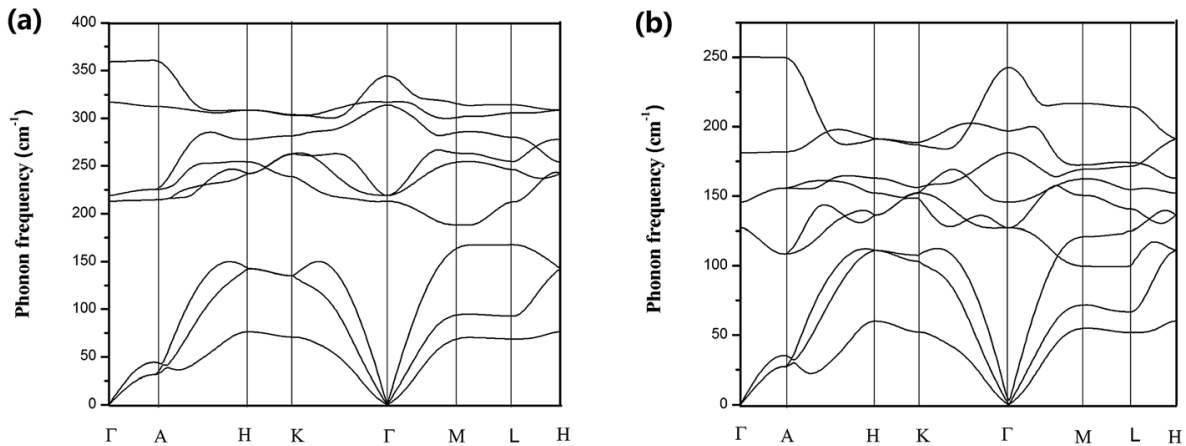

Fig. 2 Calculated phonon dispersions for (a) $\mathrm{SnS}_{2}$ and (b) $\mathrm{SnSe}_{2}$.

Table 1 Calculated Born effective charges, high-frequency dielectric tensors $\left(\varepsilon^{\infty}\right)$ and ionic contributions ( $\left.\varepsilon^{\text {ion }}\right)$ of $\mathrm{SnX}_{2}(\mathrm{X}=\mathrm{S}$ and $\mathrm{Se})$

\begin{tabular}{|c|c|c|c|c|c|}
\hline & & $Z_{\mathrm{sn}}^{*}$ & $Z_{\mathrm{x}}^{*}$ & $\varepsilon^{\infty}$ & $\varepsilon^{\text {ion }}$ \\
\hline \multirow[t]{2}{*}{$\mathrm{SnS}_{2}$} & In-plane & 4.91 & -2.50 & $9.12(8.5)^{40}$ & $9.91(10.3)^{40}$ \\
\hline & Out-of-plane & 2.08 & -1.03 & $7.51(6.7)^{40}$ & $0.69(0.6)^{40}$ \\
\hline \multirow[t]{2}{*}{$\mathrm{SnSe}_{2}$} & In-plane & 5.24 & -2.78 & 16.16 & 12.02 \\
\hline & Out-of-plane & 2.59 & -1.33 & 17.78 & 1.15 \\
\hline
\end{tabular}

In general, $\mathrm{SnS}_{2}$ and $\mathrm{SnSe}_{2}$ show very similar dispersion curves along different high symmetry lines except (1) the frequencies of the optical branches of $\mathrm{SnS}_{2}$ are significantly higher, which is mainly due to the smaller atomic mass of S. (2) There exists an evident phonon gap (about $30 \mathrm{~cm}^{-1}$ ) between the acoustic branches and optical branches for $\mathrm{SnS}_{2}$ owing to its larger cation/anion mass ratio, whereas there is no similar gap for $\mathrm{SnSe}_{2}$.

In comparison with the phonon dispersions of SnS and SnSe, ${ }^{34}$ a big difference is that the phonon spectrum of SnS and SnSe are characterized by markedly dispersive optical phonon branches, which result in significant group velocities of the optical phonon branches, whereas there are no similar phenomena for $\mathrm{SnS}_{2}$ and $\mathrm{SnSe}_{2}$. Another important feature of the phonon spectra of $\mathrm{SnX}_{2}$ is that along the $\Gamma-A$ high-symmetry $k$-paths, the phonon dispersion curves of both materials are almost flat, particularly for $\mathrm{SnS}_{2}$. This means that the phonon group velocities along the out-of-plane direction are very low, which can be attributed to ultra weak interactions between the layers. In fact, as revealed by Babu Ram et al. ${ }^{41}$ the interlayer coupling of $\mathrm{SnS}_{2}$ is much weaker than that of $\mathrm{MoS}_{2}$.

The unit cell of $\mathrm{SnX}_{2}$ contains three atoms, and therefore there are total of nine vibrational modes. Group theoretical symmetry analysis yields the irreducible representations of the $\Gamma$-point phonon modes: $\Gamma=\mathrm{A}_{1 \mathrm{~g}}+2 \mathrm{~A}_{2 \mathrm{u}}+\mathrm{E}_{\mathrm{g}}+2 \mathrm{E}_{\mathrm{u}}$. The acoustic modes are $\mathrm{A}_{2 \mathrm{u}}+\mathrm{E}_{\mathrm{u}}$. Thus, there are four optical modes, of which $\mathrm{A}_{1 \mathrm{~g}}+\mathrm{E}_{\mathrm{g}}$ are Raman active and the other two $\mathrm{A}_{2 \mathrm{u}}+\mathrm{E}_{\mathrm{u}}$ are infrared active. Note that the double degenerate $\mathrm{E}_{\mathrm{u}}$ mode has two orthogonal eigenvectors, which result in LO-TO splitting when the phonon propagates in the $a b$ plane, that is, $\mathrm{E}_{\mathrm{u}}(\mathrm{LO})$ and $\mathrm{E}_{\mathrm{u}}(\mathrm{TO})$ modes. The electrostatic interaction between the ions pushes the longitudinal optical (LO) phonons to high frequency, whereas the transversal optical (TO) phonons are unaffected. Similarly, the LO-TO splitting effect also separate the $\mathrm{A}_{2 \mathrm{u}}$ mode into $\mathrm{A}_{2 \mathrm{u}}(\mathrm{LO})$ and $\mathrm{A}_{2 \mathrm{u}}$ (TO) modes. In Table 2 we list the calculated frequencies of the optical vibrational modes considering the LO-TO splitting and compare experimentally measured Raman values since the $\mathrm{A}_{1 \mathrm{~g}}$ and $\mathrm{E}_{\mathrm{g}}$ symmetries are easily distinguished experimentally.

The specific oscillation patterns of the optical modes are shown in Fig. 3. We can find that $\mathrm{E}_{\mathrm{g}}$ and $\mathrm{E}_{\mathrm{u}}$ correspond to inplane vibration modes, whilst $\mathrm{A}_{2 \mathrm{u}}$ and $\mathrm{A}_{1 \mathrm{~g}}$ are out-of-plane vibration modes. In addition, the Raman mode $\mathrm{A}_{1 \mathrm{~g}}$ and $\mathrm{E}_{\mathrm{g}}$ species involve exclusively chalcogen atom vibrations.

\subsection{Lattice thermal conductivity}

Fig. 4 shows the temperature-dependent $\kappa_{\mathrm{L}}$ of $\mathrm{SnS}_{2}$ and $\mathrm{SnSe}_{2}$ along the in-plane ( $a$ axis) and out-of-plane ( $c$ axis) directions in comparison with the experimental measured data at $300 \mathrm{~K} \cdot{ }^{19-21}$ We can see that the lattice thermal conductivities decrease with an increase in temperature following the $T^{-1}$ relationship, which implies that the thermal conductivity is given by the anharmonic phonon-phonon interactions. In addition, the thermal conductivities of both materials are extremely anisotropic; for example, the ratio of $\kappa_{\mathrm{L}}^{\text {in-plane }} / \kappa_{\mathrm{L}}^{\text {out-plane }}$ for $\mathrm{SnSe}_{2}$ at 300 $\mathrm{K}$ is as large as 7.1, which is much higher than that of $\mathrm{MoSe}_{2}$ $(\sim 5.0)^{33}$ and SnSe $(\sim 2.5),{ }^{34}$ showing much larger anisotropy of the present materials. The large anisotropy between the inplane and out-of-plane thermal conductivity is largely due to the much smaller group velocities in the $c$ direction. This is the result of the interlayer (vdW) interactions being much weaker than the intralayer Sn-X covalent bonding. The average lattice

Table 2 Calculated frequencies of the optical vibrational modes at the Brillouin zone center $\Gamma$ point for $\mathrm{SnX}_{2}$ and comparison with other reports

\begin{tabular}{llllllll}
\hline & & $\mathrm{A}_{2 \mathrm{u}}(\mathrm{LO})$ & $\mathrm{A}_{2 \mathrm{u}}(\mathrm{TO})$ & $\mathrm{A}_{1 \mathrm{~g}}$ & $\mathrm{E}_{\mathrm{u}}(\mathrm{LO})$ & $\mathrm{E}_{\mathrm{g}}$ & $\mathrm{E}_{\mathrm{u}}(\mathrm{TO})$ \\
\hline $\mathrm{SnS}_{2}$ & Calc $^{\text {this work }}$ & 358 & 342 & 317 & 314 & 210 & 215 \\
& Calc $^{42}$ & 356 & 340 & 315 & 314 & 208 & 205 \\
& Expt $^{39}$ & & & 313.9 & & 205 & \\
$\mathrm{SnSe}_{2}$ & Calc $^{\text {this work }}$ & 250 & 242 & 183 & 199 & 126 & 145 \\
& Calc $^{42}$ & 248 & 241 & 186 & 204 & 113 & 144 \\
& Expt $^{39}$ & & & 185.9 & & 118.9 &
\end{tabular}



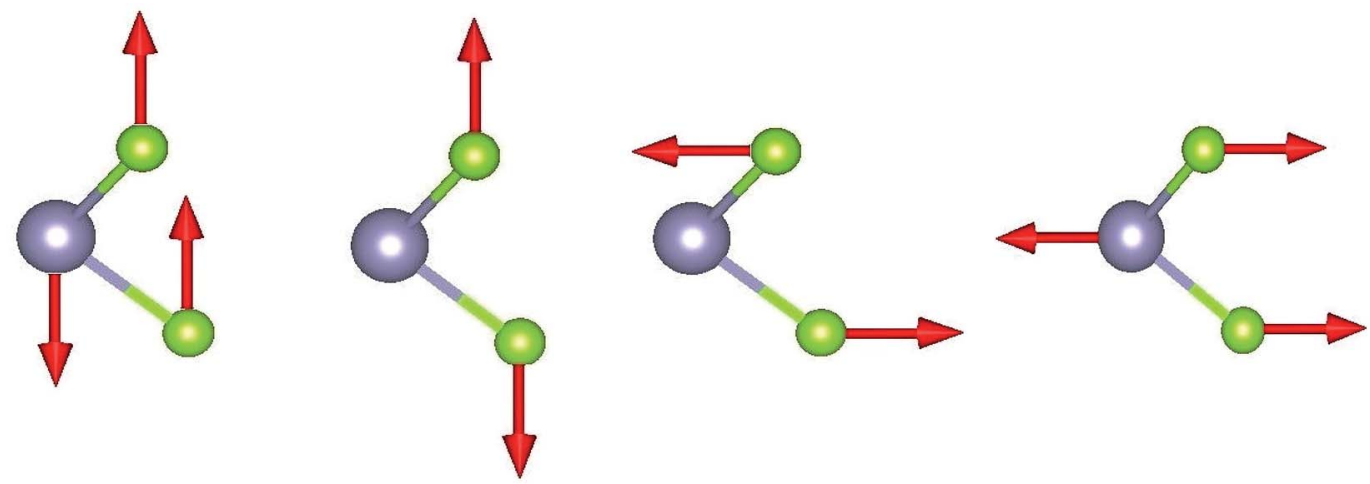

$A_{2 u}$ (IR)

$A_{\lg }(\mathrm{R})$

$E_{\mathrm{g}}(\mathrm{R})$

$E_{u}(\mathrm{IR})$

Fig. 3 Irreducible representations for $\mathrm{SnX}_{2}$ at the Brillouin zone center $\Gamma$ point.

thermal conductivity of bulk $\mathrm{SnS}_{2}$ and $\mathrm{SnSe}_{2}$ can be evaluated as $2 / 3 \kappa_{\mathrm{L}}^{\text {in-plane }}+1 / 3 \kappa_{\mathrm{L}}^{\text {out-plane }} \cdot{ }^{33}$ Our calculated average $\kappa_{\mathrm{L}}$ values at room temperature $\left(8.2 \mathrm{~W} \mathrm{mK}^{-1}\right.$ for $\mathrm{SnS}_{2}$ and $5.6 \mathrm{~W} \mathrm{mK} \mathrm{m}^{-1}$ for $\left.\mathrm{SnSe}_{2}\right)$ are close to the historically measured $\kappa_{\mathrm{L}}$ of bulk single crystals (10 $\mathrm{W} \mathrm{mK}^{-1}$ (ref. 20) for $\mathrm{SnS}_{2}$ and 7.29 $\mathrm{W} \mathrm{mK}^{-1}$ (ref. 21) for $\mathrm{SnSe}_{2}$ at $300 \mathrm{~K}$ ). Although the calculated thermal conductivity for $\mathrm{SnS}_{2}$ is much larger than the experimental value for 22 nm-thick nanosheets $\left(4.63 \mathrm{~W} \mathrm{mK}^{-1}\right){ }^{19}$ it is perfectly reasonable since in $2 \mathrm{D} \mathrm{SnS}_{2}$, the surface phonon scattering may be greatly enhanced and thus possibly decrease the lattice thermal conductivity. In comparison with other typical layer materials, the lattice thermal conductivities of $\mathrm{SnX}_{2}$ are much larger than that of $\mathrm{SnS}\left(\sim 1.6 \mathrm{~W} \mathrm{mK}^{-1}\right.$ (ref. 34$)$ at $300 \mathrm{~K}$ calculated using the same iterative self-consistent method with the ShengBTE code and the experimental value of $\sim 1.7 \mathrm{~W} \mathrm{mK}^{-1}$ (ref. 43)) and SnSe $\left(\sim 1.5 \mathrm{~W} \mathrm{mK}^{-1}\right.$ (ref. 34$)$ at $300 \mathrm{~K}$ calculated with the ShengBTE code and the experimental value of $\sim 0.67 \mathrm{~W} \mathrm{mK}^{-1}$ (ref. 24)), but greatly lower than that of $\mathrm{MoS}_{2}\left(\sim 67 \mathrm{~W} \mathrm{mK}^{-1}\right.$ (ref. 36$)$ ) at $300 \mathrm{~K}$ calculated with the ShengBTE code and the experimental value of $110 \pm 20 \mathrm{~W} \mathrm{mK}^{-1}$ (ref. 44)) and $\mathrm{MoSe}_{2}\left(\sim 60 \mathrm{~W} \mathrm{mK}^{-1}\right.$ (ref. 33) at $300 \mathrm{~K})$ calculated using the ShengBTE code.

What is even more important is that the out-of-plane thermal conductivities for both materials are found to be unexpectedly low; for example, the calculated room-temperature $\kappa_{\mathrm{L}}$ of $\mathrm{SnS}_{2}$ along the out-of-plane direction is $0.78 \mathrm{~W} \mathrm{mK}^{-1}$, which approaches the predicted amorphous limit $\left(0.47 \mathrm{~W} \mathrm{mK}^{-1}\right)^{18}$ and is even lower than that of $\mathrm{SnS}\left(0.8 \mathrm{~W} \mathrm{mK}^{-1}\right.$ along the out-ofplane direction at room temperature). ${ }^{34}$ Furthermore, these values are remarkably low among most of the TMDs; for example, the calculated out-of-plane thermal conductivity using the same method for $\mathrm{MoS}_{2}$ is $2.3 \mathrm{~W} \mathrm{mK}{ }^{-1}$ (ref. 36) (the experimental out-of-plane thermal conductivity of bulk $\mathrm{MoS}_{2}$ at $300 \mathrm{~K}$ is $1.05 \mathrm{~W} \mathrm{mK}^{-1}$ (ref. 45)) and that of $\mathrm{WTe}_{2}$ is $1.04 \mathrm{~W} \mathrm{mK}^{-1} .^{38}$ This implies that the thermoelectric performance of $\mathrm{SnS}_{2}$ along the out-of-plane direction may be as good as that of SnS. In fact, it is difficult to definitely say how its thermoelectric performance would be because the thermoelectric figure of merit is determined by several strongly coupled quantities and the lattice thermal conductivity is only one of them, but at least, we can conclude that the present material is a good thermal insulator in the out-of-plane direction. Unfortunately, the experimentally measured thermal conductivity values of the out-of-plane direction are not yet available in the literature.

Using the obtained thermal conductivity values of $\mathrm{SnX}_{2}$, its thermal diffusivity can be derived from the formula $\alpha=\kappa_{\mathrm{L}} / C_{\mathrm{p}} \rho$ ( $\kappa_{\mathrm{L}}$ is thermal conductivity, $C_{\mathrm{p}}$ is heat capacity and $\rho$ is density).
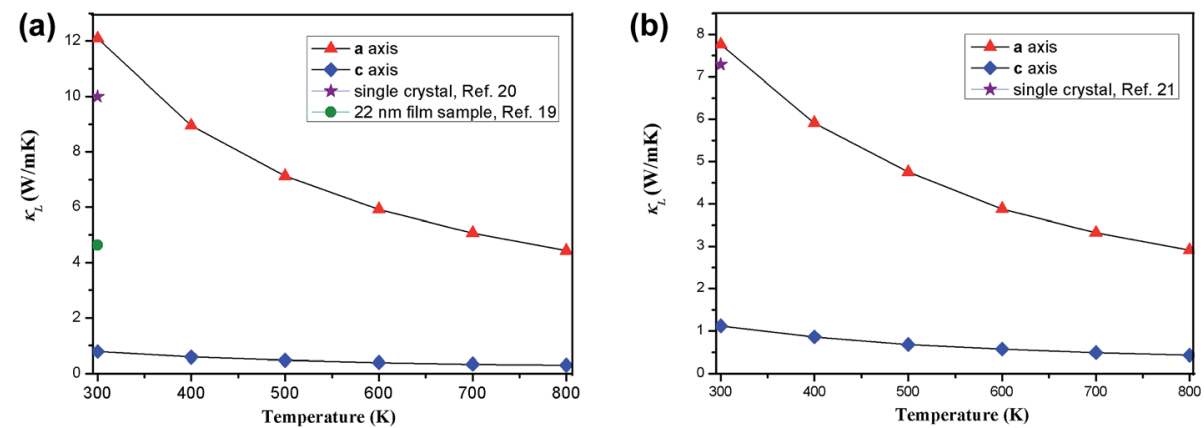

Fig. 4 Calculated temperature dependent lattice thermal conductivities of (a) $\mathrm{SnS}_{2}$ and (b) $\mathrm{SnSe}_{2}$ along the $a$ axis and $c$ axis, in comparison with the experimental data at $300 \mathrm{~K}$. 
Table 3 Contributions from phonon branches to the total thermal conductivity for $\mathrm{SnX}_{2}$ at $300 \mathrm{~K}$

\begin{tabular}{llllll}
\hline & & TA1 & TA2 & LA & Optic \\
\hline $\mathrm{SnS}_{2}$ & In-plane & $16 \%$ & $35 \%$ & $43 \%$ & $6 \%$ \\
& Out-of-plane & $30 \%$ & $24 \%$ & $24 \%$ & $22 \%$ \\
$\mathrm{SnSe}_{2}$ & In-plane & $14 \%$ & $36 \%$ & $41 \%$ & $9 \%$ \\
& Out-of-plane & $28 \%$ & $22 \%$ & $35 \%$ & $15 \%$
\end{tabular}

Thermal diffusivity is one of the crucial parameters to investigate the thermal transportation process, which indicates that the rate of temperature changes and reflects the correlation between thermal conductivity and heat capacity. ${ }^{46-49}$ The TAPP software $^{50}$ gives heat capacity values of $C_{\mathrm{p}}^{\mathrm{s}}=252 \mathrm{~J} \mathrm{~kg} \mathrm{~K}^{-1}$ for $\mathrm{SnS}_{2}$ and $C_{\mathrm{p}}^{\mathrm{Se}}=261 \mathrm{~J} \mathrm{~kg} \mathrm{~K}^{-1}$ for $\mathrm{SnSe}_{2}$ at room temperature. The density of $\mathrm{SnX}_{2}$ is easily calculated as $\rho_{\mathrm{S}}=4700 \mathrm{~kg} \mathrm{~m}^{-3}$ for $\mathrm{SnS}_{2}$ and $\rho_{\mathrm{Se}}=6200 \mathrm{~kg} \mathrm{~m}^{-3}$. Then, we derive the thermal diffusivity $\alpha_{\mathrm{S}}=6.9 \times 10^{-6} \mathrm{~m}^{2} \mathrm{~s}^{-1}$ for $\mathrm{SnS}_{2}$ and $\alpha_{\mathrm{Se}}=3.4 \times 10^{-6} \mathrm{~m}^{2} \mathrm{~s}^{-1}$ for $\mathrm{SnSe}_{2}$. Clearly, the thermal diffusivity of $\mathrm{SnS}_{2}$ is much larger than that of $\mathrm{SnSe}_{2}$ mainly due to its larger thermal conductivity and smaller mass density. Our calculated thermal diffusivity for $\mathrm{SnSe}_{2}$ is close to the historically calculated value $\left(\alpha_{\mathrm{Se}}=5.4 \times\right.$ $10^{-6} \mathrm{~m}^{2} \mathrm{~s}^{-1}$ at $\left.300 \mathrm{~K}\right) ;{ }^{22}$ however, the thermal diffusivity of bulk $\mathrm{SnS}_{2}$ was not found in the literature.

We then determined the contribution of the different phonon modes to the total lattice thermal conductivities for the two materials, as illustrated in Table 3. It is found that along the in-plane direction, the contribution of the optical branches to the total thermal conductivity at room temperature for both $\mathrm{SnS}_{2}$ and $\mathrm{SnSe}_{2}$ is small, whereas along the out-of-plane direction, the contribution of the optical branches is relatively large, particularly for $\mathrm{SnS}_{2}$, where the contribution of the optical branches is as large as $22 \%$. This may be one of the reasons for the ultralow out-of-plane thermal conductivity since the optical branches usually possess much smaller group velocities and much shorter phonon lifetimes than those of the acoustic branches. The small contributions of the optical phonons of $\mathrm{SnX}_{2}$ is an obvious difference with $\mathrm{SnX}$, for instance, the optical phonon branches contribute $\sim 60 \%$ to the total thermal conductivity of SnSe. $^{34}$ Therefore, alloying may not be an effective strategy for further reducing the $\kappa_{\mathrm{L}}$ of $\mathrm{SnX}_{2}$.
Furthermore, the temperature dependence of contributions from the phonon branches is plotted in Fig. 5 using $\mathrm{SnSe}_{2}$ as an example. One can see that the contributions of all the phonon modes along the in-plane direction are very insensitive to temperature, where the percentage contributions of the acoustic phonon branches slightly decrease with an increase in temperature, and at the same time, the contribution of the optical phonon branches slightly increases with an increase in temperature. While along the out-of-plane direction, the increase tendency of the contributions of the optical branches becomes evident and reaches $16.5 \%$ at $800 \mathrm{~K}$, indicating that higher frequency optical phonons are excited at higher temperatures.

Considering the significant difference in the contribution of different phonon branches to the total thermal conductivity in $\mathrm{SnX}_{2}$, it is worthwhile to examine the relaxation times of the phonon modes as functions of phonon frequency, as displayed in Fig. 6. It is found that the relaxation times of the phonon modes for $\mathrm{SnS}_{2}$ and $\mathrm{SnSe}_{2}$ are very close to each other. Otherwise, the relaxation times of the acoustic branch are one order of magnitude bigger than that of the optical branches, which indicates small contributions of optical phonons to the total $\kappa_{\mathrm{L}}$. In comparison with $\mathrm{SnX}$ and $\mathrm{MoX}_{2}$, the relaxation times of $\mathrm{SnX}_{2}$ are much shorter than that of $\mathrm{MoX}_{2}$, although slightly longer than that of SnX. For example, the calculated longest relaxation time of acoustic phonons for $\mathrm{SnS}_{2}$ is about $160 \mathrm{ps}$, whilst that of $\mathrm{MoS}_{2}$ is as large as $10^{4} \mathrm{ps}^{36}$ and that of $\mathrm{SnS}$ is only about $28 \mathrm{ps}^{34}$

Since the phonon lifetimes of these two materials are comparable, what is the main mechanism that leads to the higher $\kappa_{\mathrm{L}}$ in $\mathrm{SnS}_{2}$ ? We next calculated the phonon group velocities of $\mathrm{SnX}_{2}$ around the $\Gamma$ point. Our calculated sound velocity in the long-wavelength limit of $\mathrm{SnS}_{2}$ is about $5060 \mathrm{~m} \mathrm{~s}^{-1}$ along the in-plane direction, and that of $\mathrm{SnSe}_{2}$ is only $4050 \mathrm{~m}$ $\mathrm{s}^{-1}$. Thus, the conclusion is clear that it is just the relatively larger phonon group velocities combined with the higher phonon frequencies of $\mathrm{SnS}_{2}$ that induce the much higher $\kappa_{\mathrm{L}}$ than $\mathrm{SnSe}_{2}$. We also evaluated the anharmonic phase space volume $P_{3}$, that is, the number of allowed three-phonon processes of the present two compounds, which is usually used to quantify the anharmonicity of a certain material. The obtained $P_{3}$ of $\mathrm{SnS}_{2}$ is $0.54 \times 10^{-2} \mathrm{eV}^{-1}$, and that of $\mathrm{SnSe}_{2}$ is
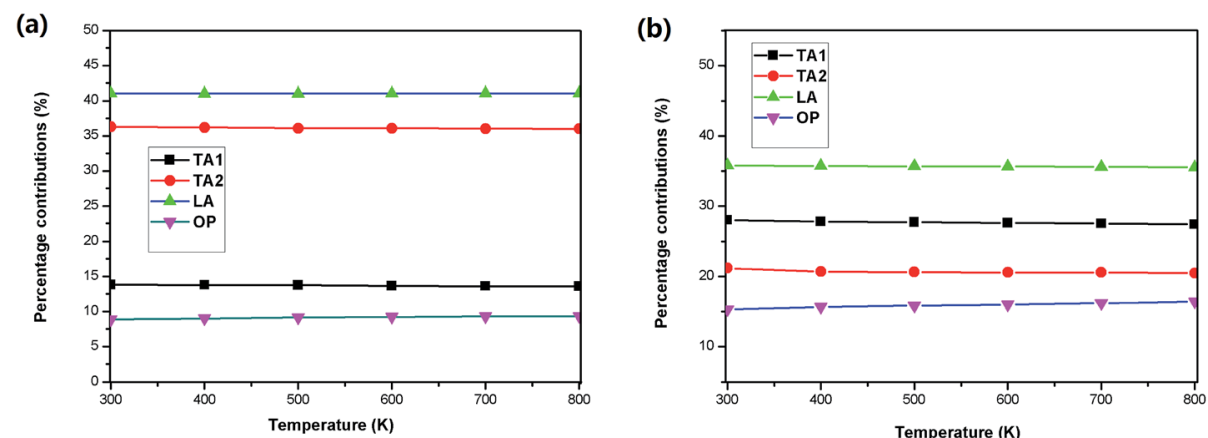

Fig. 5 Temperature dependence of the percentage contributions of the phonon branches to the total lattice thermal conductivity for SnSe 2 along the (a) in-plane direction and (b) out-of-plane direction. 
(a)

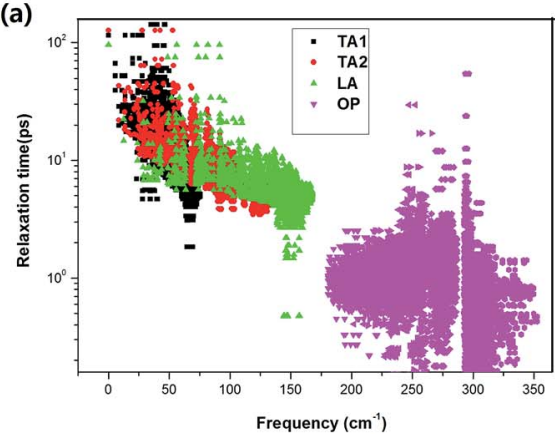

(b)

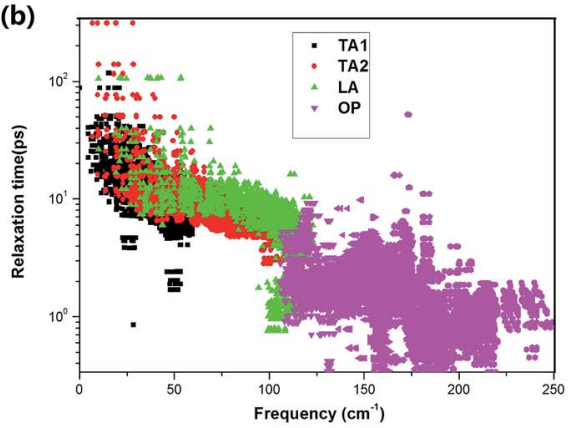

Fig. 6 Frequency-dependent phonon relaxation times of the acoustic (AP) and optical phonons (OP) for (a) $\mathrm{SnS}_{2}$ and (b) $\mathrm{SnSe}_{2}$ at $300 \mathrm{~K}$.

$0.83 \times 10^{-2} \mathrm{eV}^{-1}$; therefore it is clear that the anharmonicity of $\mathrm{SnSe}_{2}$ is higher than that of $\mathrm{SnS}_{2}$. In comparison with other lowconductivity compounds, the anharmonic phase space volume $P_{3}$ is slightly larger than that of Si $\left(0.47 \times 10^{-2} \mathrm{eV}^{-1}\right),{ }^{32}$ but much smaller than that of SnSe $\left(1.9 \times 10^{-2} \mathrm{eV}^{-1}\right) \cdot{ }^{32}$ Furthermore, the mode Grüneisen parameters are calculated as 1.18 for $\mathrm{SnS}_{2}$ and 1.21 for $\mathrm{SnSe}_{2}$, which also indicates that the anharmonicity of $\mathrm{SnSe}_{2}$ is higher than that of $\mathrm{SnS}_{2}$.

Finally, we investigate the size effects of the present materials by studying the cumulative thermal conductivity $\kappa(l)$ as a function of phonon mean free path, which is defined as the thermal conductivity contributed by phonons whose mean free path $l$ is smaller than a given value $l_{\text {max }}$. Fig. 7 shows the cumulative thermal conductivity along the $a$ and $c$ directions with respect to the phonon MPFs at room temperature, $300 \mathrm{~K}$. It can be seen that the cumulative thermal conductivity of both materials continuously increases as MFP increases, until reaching the limit length $L$, which represents the longest mean free path of the heat carriers. We derived the limit lengths of $L_{\mathrm{a}} \sim 585 \mathrm{~nm}$ and $L_{\mathrm{c}} \sim$ $400 \mathrm{~nm}$ for $\mathrm{SnS}_{2}$, and $L_{\mathrm{a}} \sim 575 \mathrm{~nm}$ and $L_{\mathrm{c}} \sim 1100 \mathrm{~nm}$ for $\mathrm{SnSe}_{2}$. It should be noted that the limit lengths $L$ of the present compounds are much larger than that of SnS and SnSe. ${ }^{32,34}$

Then, we fitted the cumulative $\kappa(l)$ for $\mathrm{SnX}_{2}$ along the inplane and out-of-plane directions at $300 \mathrm{~K}$ to a single parametric function, ${ }^{51,52} \kappa\left(l<l_{\max }\right)=\frac{\kappa_{\max }}{1+l_{0} / l_{\max }}$, where, $\kappa_{\max }$ is the ultimate cumulated $\kappa, l_{\max }$ is the maximal MFP concerned, and $l_{0}$ is the parameter to be evaluated by the fitting. The fitted curves are plotted in Fig. 7, which reproduce the calculated data well and yield the parameter $l_{0}$ for the two materials along the in-plane and out-of-plane directions, which could be interpreted as the representative MFP (rMFP). The rMFP is helpful to study the size effect on ballistic or diffusive phonon transport, which is important for thermal design with nanostructuring. Our fitted rMFPs $\left(l_{0}\right)$ for $\mathrm{SnS}_{2}$ are $30 \mathrm{~nm}$ along the in-plane direction and $20 \mathrm{~nm}$ along the out-of-plane direction, and for $\mathrm{SnSe}_{2}$, they are $24 \mathrm{~nm}$ along the in-plane direction and $38 \mathrm{~nm}$ along the out-of-plane direction. These values are quite larger than that of SnSe $(4.30 \mathrm{~nm}$ along the armchair direction and $6.86 \mathrm{~nm}$ along the zigzag direction). ${ }^{35}$ Such large representative rMFPs suggest that there are strong size effects in the phonon transport of micro-/nano-sized materials based on these two materials. Our results suggest that to reduce the lattice thermal conductivities of both materials, nanostructuring will be an effective way. This suggestion is consistent with recent experiment findings, where the $\kappa_{\mathrm{L}}$ of $\mathrm{SnS}_{2}$ was found to evidently decrease with a decrease in the thickness of $\mathrm{SnS}_{2}$ films. ${ }^{19}$ Moreover, we believe that such a phenomenon may also exist in $\mathrm{SnSe}_{2}$, that is, this material may also be a negatively correlated thermoelectric material just like $\mathrm{SnS}_{2}$ with a negative correlation between electrical and thermal conductivity. In addition, $\mathrm{SnSe}_{2}$ may demonstrate a much better thermoelectric performance than that of $\mathrm{SnS}_{2}$ because of its higher anharmonicity than that of $\mathrm{SnS}_{2}$, leading to much lower thermal conductivity.
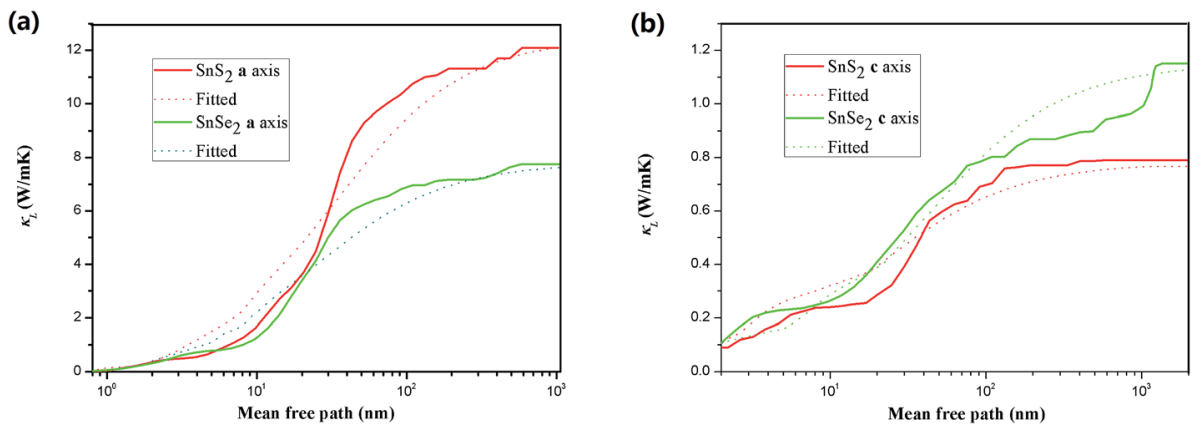

Fig. 7 Cumulative lattice thermal conductivity of $\mathrm{SnX}_{2}$ along the (a) in-plane and (b) out-of-plane directions as a function of phonon MFP at room temperature. The fitted curves are plotted with dotted lines. 


\section{Conclusion}

In summary, we have systematically investigated the anisotropic phonon transport properties and intrinsic lattice thermal conductivities for tin dichalcogenides $\left(\mathrm{SnS}_{2}\right.$ and $\mathrm{SnSe}_{2}$ ) from first principles calculations. The calculated inplane lattice thermal conductivity of $\mathrm{SnS}_{2}$ is $12.10 \mathrm{~W} \mathrm{mK} \mathrm{mK}^{-1}$, whereas that of $\mathrm{SnSe}_{2}$ is much lower $\left(7.76 \mathrm{~W} \mathrm{mK}{ }^{-1}\right)$, which mainly originates from its relatively smaller phonon group velocities combined with lower phonon frequencies. The outof-plane thermal conductivities of both materials are found to be remarkably low $\left(0.78 \mathrm{~W} \mathrm{mK}^{-1}\right.$ for $\mathrm{SnS}_{2}$ and $1.12 \mathrm{~W} \mathrm{mK}^{-1}$ for $\mathrm{SnSe}_{2}$ ) due to very weak interlayer forces, which indicates their significance in thermoelectric applications. The obtained total thermal conductivities of $\mathrm{SnS}_{2}$ and $\mathrm{SnSe}_{2}$ agree well with experimental measured values. The phonon lifetimes of all branches are found to be much shorter in comparison with that of $\mathrm{MoS}_{2}\left(\mathrm{MoSe}_{2}\right)$ but slightly longer than that of $\mathrm{SnS}$ (SnSe). The contributions of the optical phonon branches to the total thermal conductivities are found to be rather small, which suggests that it would be difficult for the strategy of alloying to reduce $\kappa_{\mathrm{L}}$. However, nanostructuring may be effective to further reduce $\kappa_{\mathrm{L}}$ since the mean free paths of the dominant phonon modes are relatively long. This conclusion is consistent with recent experiment findings, where the $\kappa_{\mathrm{L}}$ of $\mathrm{SnS}_{2}$ thin films were found to be much lower than that of bulk $\mathrm{SnS}_{2}$. Our study not only presents comprehensive investigations on the phonon transport properties of $\mathrm{SnS}_{2}$ and $\mathrm{SnSe}_{2}$, but also provides discussions and analyses on the origins of the diverse heat transfer phenomena, which would be of significance for further studies and applications based on these two TMDs.

\section{Acknowledgements}

This study was supported by the Natural Science Foundation of China (Grant no. 11145004 and 21363019).

\section{Notes and references}

1 J. Gu, X. Yang, Z. Lv, N. Li, C. Liang and Q. Zhang, Int. J. Heat Mass Transfer, 2016, 92, 15-22.

2 J. Gu, C. Xie, H. Li, J. Dang, W. Geng and Q. Zhang, Polym. Compos., 2014, 35, 1087-1092.

3 J. Gu, X. Meng, Y. Tang, Y. Li, Q. Zhuang and J. Kong, Composites, Part A, 2017, 92, 27-32.

4 J. Gu, C. Liang, J. Dang, W. Dong and Q. Zhang, RSC Adv., 2016, 6, 35809-35814.

5 S. Gedi, V. R. M. Reddy, B. Pejjai, C.-W. Jeon and C. Park, Appl. Surf. Sci., 2016, 372, 116-124.

6 X. Jing, D. D. Zhu, L. Wang, B. Huang, X. Huang and X. M. Meng, Adv. Funct. Mater., 2015, 25, 4255-4261.

7 X. Zhou, Q. Zhang, L. Gan, H. Li and T. Y. Zhai, Adv. Funct. Mater., 2016, 26, 4405-4413.

8 S. Song, S. L. Li, L. Gao, Y. Xu, K. Ueno, J. Tang, Y. B. Chengad and K. Tsukagoshi, Nanoscale, 2013, 5, 9666-9670.
9 F. Zhang, C. Xia, J. Zhu, B. Ahmed, H. Liang, D. B. Velusamy, U. Schwingenschlögl and H. N. Alshareef, Adv. Energy Mater., 2016, 16, 01188.

10 C. KyungMin, D. Wamwangi, M. Woda, M. Wuttig and W. Bensch, J. Appl. Phys., 2008, 103, 083523.

11 Z. Sun and H. Chang, ACS Nano, 2014, 8, 4133-4156.

12 H. Zhong, G. Yang, H. Song, Q. Liao, H. Cui, P. Shen and

C. Wang, J. Phys. Chem. C, 2012, 116, 9319-9326.

13 X. He and H. Shen, Phys. B, 2012, 407, 1146-1152.

14 L. A. Burton, D. Colombara, R. D. Abellon, F. C. Grozema, L. M. Peter, T. J. Savenije, G. Dennler and A. Walsh, Chem. Mater., 2013, 25, 4908-4916.

15 S. Mandalidis, J. A. Kalomiros, K. Kambas and A. N. Anagnostopoulos, J. Mater. Sci., 1996, 31, 5975-5978.

16 A. Voznyi, V. Kosyak, A. Opanasyuk, N. Tirkusova, L. Grase, A. Medvids and G. Mezinskis, Mater. Chem. Phys., 2016, 173, 52-61.

17 A. A. Kozma, M. Y. Sabov, E. Y. Peresh, I. E. Barchiy and V. V. Tsygyka, Inorg. Mater., 2015, 51, 93-97.

18 B. Sun, Z. Ma, C. Hea and K. Wu, Phys. Chem. Chem. Phys., 2015, 17, 29844-29853.

19 M. J. Lee, J. H. Ahn, J. H. Sung, H. Heo, S. G. Jeon, W. Lee, J. Y. Song, K. H. Hong, B. Choi, S. H. Lee and M. H. Jo, Nat. Commun., 2016, 7, 12011.

20 C. Khélia, F. Maïz, M. Mnari, T. Ben Nasrallah, M. Amlouk and S. Belgacem, Eur. Phys. J.: Appl. Phys., 2000, 9, 187-193.

21 G. Busch, C. Fröhlich, F. Hulliger and E. Steigmeier, Helv. Phys. Acta, 1961, 34, 359.

22 Z. V. Borges, C. M. Poffo, J. C. de Lima, S. M. de Souza, D. M. Triches, T. P. O. Nogueira, L. Manzato and R. S. de Biasi, Mater. Chem. Phys., 2016, 169, 47-54.

23 A. A. Kozma, M. Yu. Sabov, E. Yu. Peresh, I. E. Barchiy and V. V. Tsygyka, Inorg. Mater., 2015, 51, 93-97.

24 L.-D. Zhao, S.-H. Lo, Y. Zhang, H. Sun, G. Tan, C. Uher, C. Wolverton, V. P. Dravid and M. G. Kanatzidis, Nature, 2014, 508, 373-377.

25 L.-D. Zhao, G. Tan, S. Hao, J. He, Y. Pei, H. Chi, H. Wang, S. Gong, H. Xu and V. P. Dravid, Science, 2016, 351, 141-144. 26 G. Kresse and J. Furthmüller, Comput. Mater. Sci., 1996, 6, 15. 27 G. Kresse and J. Furthmüller, Phys. Rev. B: Condens. Matter Mater. Phys., 1996, 54, 11169.

28 G. Kresse and D. Joubert, Phys. Rev. B: Condens. Matter Mater. Phys., 1999, 59, 1758.

29 J. P. Perdew and A. Zunger, Phys. Rev. B: Condens. Matter Mater. Phys., 1981, 23, 5048.

30 A. Togo, F. Oba and I. Tanaka, Phys. Rev. B: Condens. Matter Mater. Phys., 2008, 78, 134106.

31 W. Li, J. Carrete, N. A. Katcho and N. Mingo, Comput. Phys. Commun., 2014, 185, 1747.

32 J. Carrete, N. Mingo and S. Curtarolo, Appl. Phys. Lett., 2014, 105, 101907.

33 S. Kumar and U. Schwingenschlögl, Chem. Mater., 2015, 27, 1278-1284.

34 R. Guo, X. Wang, Y. Kuang and B. Huang, Phys. Rev. B: Condens. Matter Mater. Phys., 2015, 92, 115202.

35 G. Qin, Z. Qin, W.-Z. Fang, L.-C. Zhang, S.-Y. Yue, Q.-B. Yan, M. Hu and G. Su, Nanoscale, 2016, 8, 11306-11319. 
36 A. N. Gandi and U. Schwingenschögl, EPL, 2016, 113, 36002. 37 B. Peng, H. Zhang, H. Shao, Y. Xu, X. Zhang and H. Zhu, Ann. Phys., 2016, 528, 504-511.

38 G. Liu, H. Y. Sun, J. Zhou, Q. F. Li and X. G. Wan, New J. Phys., 2016, 18, 033017.

39 V. G. Hadjiev, D. De, H. B. Peng, J. Manongdo and A. M. Guloy, Phys. Rev. B: Condens. Matter Mater. Phys., 2013, 87, 104302.

40 Y. Kumagai, L. A. Burton, A. Walsh and F. Oba, Phys. Rev. Appl., 2016, 6, 014009.

41 B. Ram, A. Manjanath and A. K. Singh, 2D Mater., 2016, 3, 015009.

42 G. Lucovsky, J. C. Mikkelslen Jr., W. Y. Liang and R. M. White, Phys. Rev. B: Solid State, 1976, 14, 1663-1669.

43 J. Wasscher, W. Albers and C. Haas, Solid-State Electron., 1963, 6, 261.

44 J. Liu, G. M. Choi and D. G. Cahill, J. Appl. Phys., 2014, 116, 233107.
45 H. J. Goldsmid, in Semiconductors and Semimetals, ed. T. M. Tritt, Elsevier, 2001, vol. 69, p. 1.

46 V. Kishore, R. Sharma, V. K. Saraswat, N. S. Saxena, K. Sharma and T. P. Sharma, Appl. Therm. Eng., 2007, 27, 1552-1554.

47 J. M. Laskar, S. Bagavathiappan, M. Sardar, T. Jayakumar, J. Philip and B. Raj, Mater. Lett., 2008, 62, 2740-2742.

48 K. Zhou and B. Wei, Appl. Phys. A: Mater. Sci. Process., 2016, $122,248$.

49 W. Zhai, K. Zhou, L. Hu and B. Wei, J. Chem. Thermodyn., 2016, 95, 159-163.

50 O. H. Hamilton, TAPP Software Version 2.2, E. S. Microwave Inc, Wade Court, 1990.

51 W. Li, J. Carrete, N. A. Katcho and N. Mingo, Comput. Phys. Commun., 2014, 185, 1747-1758.

52 G. Qin, Q.-B. Yan, Z. Qin, S.-Y. Yue, M. Hu and G. Su, Phys. Chem. Chem. Phys., 2015, 17, 4854. 